\title{
Determination of the Strong Coupling Constant by the ALPHA Collaboration
}

\author{
Tomasz Korzec ${ }^{1, \star}$ for the ALPHA collaboration \\ ${ }^{1}$ Department of Physics, Bergische Universität Wuppertal, Gaußstr. 20, 42119 Wuppertal, Germany
}

\begin{abstract}
A high precision determination of the strong coupling constant in the $\overline{\mathrm{MS}}$ scheme at the Z-mass scale, using low energy quantities, namely pion/kaon decay constants and masses, as experimental input is presented. The computation employs two different massless finite volume renormalization schemes to non-perturbatively trace the scale dependence of the respective running couplings from a scale of about $200 \mathrm{MeV}$ to $100 \mathrm{GeV}$. At the largest energies perturbation theory is reliable. At high energies the Schrödinger-Functional scheme is used, while the running at low and intermediate energies is computed in a novel renormalization scheme based on an improved gradient flow. Large volume $N_{\mathrm{f}}=2+1 \mathrm{QCD}$ simulations by CLS are used to set the overall scale. The result is compared to world averages by FLAG and the PDG.
\end{abstract}

\section{Introduction}

Parameters of the standard model have to be determined experimentally before any predictions can be made. Improvements in the knowledge of these fundamental quantities translate into a higher predictive power of the model and are crucial for the successful operation of expensive collider experiments like the LHC. A parameter that is most important and so far not particularly precisely determined is the coupling in the strong sector of the standard model. The difficulties in its determination lie in the confining properties of QCD. The typical method to measure it is to "fit" the perturbative prediction of a high energy process to the corresponding measurement. In order for the truncated perturbative series to describe the process well, one is forced to concentrate on processes where the physical energy scale of the process is high, i.e. $\mu \gg 1 \mathrm{GeV}$. Only then the QCD coupling is small enough and truncation errors are under control. The main uncertainties are systematic errors associated with the necessary processing of the raw data, before it can be compared to perturbation theory. To infer what fundamental process has taken place from the measured energies and momenta of photons, leptons and hadrons, one relies not only on a detailed mathematical model of the detector, but also on a good understanding of the hadronization process. The latter is too complicated to be computed within the standard model and various model assumptions enter which in the end may dominate the systematic error. Furthermore many experiments rely on a set of measured structure functions, which can introduce subtle correlations in the results of different collaborations.

A summary of the methods and most precise experimental determinations is compiled regularly by the particle data group [1]. The different results are combined into a world average, its most recent

^ Speaker, e-mail: korzec@uni-wuppertal.de 

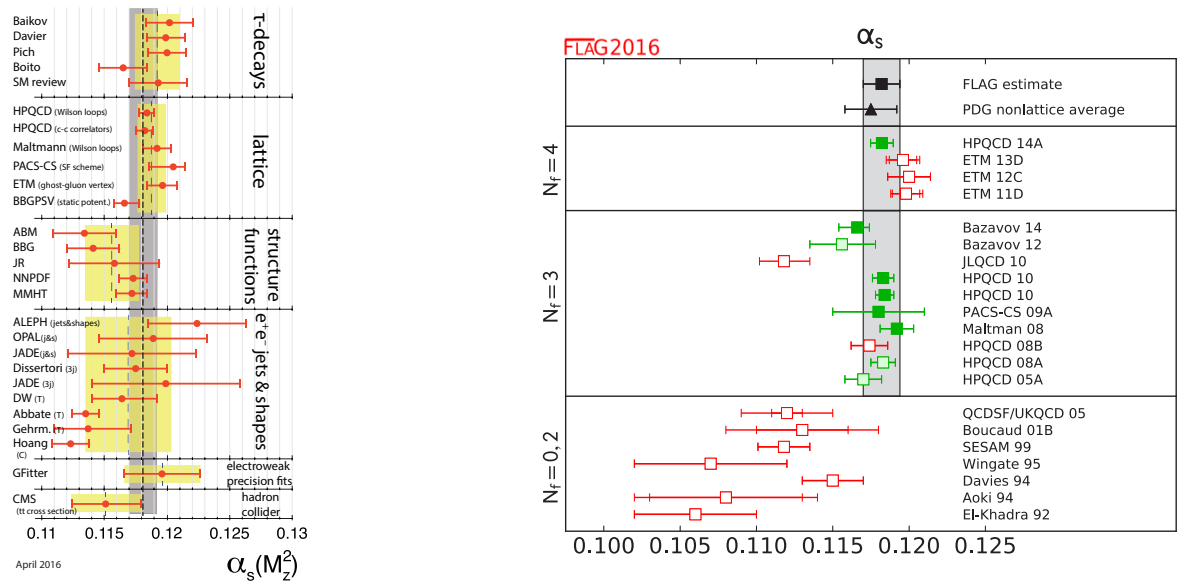

Figure 1. The left panel is PDG's [1] summary of $\alpha_{\mathrm{S}}$ determinations. The yellow bands correspond to the preaverages of different classes of determinations. The right panel, by FLAG [2], focuses on determinations that used lattice QCD as a tool. The gray bands are the global averages of the two groups.

value being $\alpha_{\mathrm{S}}\left(M_{\mathrm{Z}}\right)=0.1181(11)$. This is the $\overline{\mathrm{MS}}$-coupling of the five flavor theory renormalized at $\mu=M_{\mathrm{Z}}, \alpha_{\mathrm{S}}(\mu) \equiv\left(\bar{g}_{\overline{\mathrm{MS}}}^{(5)}(\mu)\right)^{2} /(4 \pi)$. In recent years lattice QCD has become increasingly important as a tool that allows to connect low and high energy regimes non-perturbatively. The coupling $\alpha_{\mathrm{S}}\left(M_{\mathrm{Z}}\right)$ can then be determined from measured values of low energy hadronic quantities like pion masses and decay constants. Currently some of the world's most precise determinations are based on lattice QCD and already dominate the world average. The latest status is summarized in Fig. 1. Lattice determinations are also reviewed and averaged by the Flavour Lattice Averaging Group [2]. The current FLAG average includes results from [3-7]. Most of the uncertainties in these lattice determinations are systematic in nature and are rooted in the multi-scale nature of the problem. On the one hand the spatial extent $L$ of the simulated box needs to be large enough to avoid finite volume effects in hadronic observables, i.e. $L \gg m_{\pi}^{-1}$. On the other hand the lattice spacing $a$ must be fine enough to be able to compute a renormalized coupling at high energies $\mu$ (where it is small). A coupling could for instance be defined through the static force at short distances $r=\mu^{-1}$, which would require $a \ll \mu^{-1}$. Insisting on a high value of $\mu$, e.g. $\mu \approx 100 \mathrm{GeV}$ immediately leads to astronomically large lattice sizes $L / a$. With today's machines and algorithms one is restricted to $L / a \lesssim 100$ which requires a careful balance of the scales $a, \mu$ and $L$ such that the unavoidable finite size-, cutoff- and perturbative truncation errors are all under control. For instance the most recent result [7] of Fig. 1 uses lattices with up to $L / a=64$ sites, renormalization scales $\mu \approx 5 \mathrm{GeV}$ with lattice spacings $a^{-1} \approx 3.3 \mathrm{GeV}$ and coarser.

These systematic errors can be nearly eliminated by switching to finite volume renormalization schemes [8], where $\mu \equiv L^{-1}$. The drawback is, that a whole sequence of simulations at various values of $L$ becomes necessary. In addition, the results based on finite size scaling were so far plagued by relatively large statistical errors or an insufficient number of dynamical flavors. The only calculation of this type that enters the FLAG average is the one by PACS-CS, [4]. It has a large, but mainly statistical error. 
This article summarizes the effort of the ALPHA collaboration to determine $\alpha_{\mathrm{S}}$ using finite-sizescaling techniques. It is self-contained but, due to size restrictions, many details are omitted. The reader is encouraged to consult the original literature [9-13] for a deeper understanding of the calculation.

The outline of this proceeding contribution is as follows: in Sec. 2 our notation is fixed and basic formulae collected. Sec. 3 lays out our computational strategy and the different more or less independent parts of the calculation are treated in some detail in sections 4 - 6 . The article concludes with Sec. 7 where the final result is put together and discussed.

\section{Renormalization Schemes and Scales}

Almost any renormalized, dimensionless, short-distance quantity $\phi$ can be the starting point for the definition of a renormalization scheme. If it possesses a perturbative expansion in a bare coupling $g_{0}$, e.g. $\phi=\sum_{l} \phi_{l} g_{0}^{2 l}$, one can define a renormalized coupling in this scheme as $\bar{g}_{\phi}^{2} \equiv \frac{\phi-\phi_{0}}{\phi_{1}}$. The quantity $\phi$, the coefficients $\phi_{l}$ and therefore the renormalized coupling depend on a scale $\mu$. The scale dependence of the coupling is expressed by the RG equation

$$
\mu \frac{d \bar{g}}{d \mu}=\beta(\bar{g}) .
$$

The $\beta$ function depends on the scheme, but in massless schemes the first two coefficients in a perturbative expansion

$$
\beta(\bar{g})=-\bar{g}^{3}\left(b_{0}+b_{1} \bar{g}^{2}+b_{2} \bar{g}^{4}+\ldots\right)
$$

are "universal"

$$
b_{0}=\frac{1}{16 \pi^{2}}\left(11-\frac{2 N_{\mathrm{f}}}{3}\right), \quad b_{1}=\frac{1}{256 \pi^{4}}\left(102-\frac{38 N_{\mathrm{f}}}{3}\right) .
$$

The subsequent coefficients depend on the scheme. They are known to five loops $\left(b_{2}\right.$ through $\left.b_{4}\right)$ in the $\overline{\mathrm{MS}}$-scheme [14-18] and to three loops $\left(b_{2}\right)$ in the SF-scheme [19]. The solution of the ODE Eq. (2) requires an initial condition, or equivalently, the introduction of a $\Lambda$-parameter, e.g. at one loop ${ }^{1}$

$$
\bar{g}^{2}(\mu)=\frac{1}{2 b_{0} \ln (\mu / \Lambda)} \quad \text { or } \quad \Lambda=\mu e^{-1 /\left(2 b_{0} \bar{g}^{2}(\mu)\right)}
$$

The well known fully non-perturbative expression is

$$
\Lambda=\mu\left(b_{0} \bar{g}^{2}(\mu)\right)^{-\frac{b_{1}}{2 b_{0}^{2}}} \exp \left[-\frac{1}{2 b_{0} \bar{g}^{2}(\mu)}-\int_{0}^{\bar{g}(\mu)}\left(\frac{1}{\beta(x)}+\frac{1}{b_{0} x^{3}}-\frac{b_{1}}{b_{0}^{2} x}\right) d x\right] .
$$

$\Lambda$ parameters are scheme dependent. Between two schemes with couplings $\bar{g}$ and $\bar{g}^{\prime}$ that are related in perturbation theory by

$$
\bar{g}^{\prime 2}(\mu)=\bar{g}^{2}(\mu)+c \bar{g}^{4}(\mu)+\ldots
$$

the exact relation between $\Lambda$ parameters is

$$
\Lambda^{\prime}=\Lambda e^{c /\left(2 b_{0}\right)} .
$$

\footnotetext{
${ }^{1}$ Eq. (4) is more of pedagogical than practical value, since it cannot be used to determine $\Lambda$, no matter how high $\mu$ is. It does

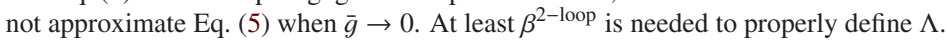


Instead of the $\beta$-function, the step-scaling function $\sigma\left(\bar{g}^{2}\right)$ can be invoked to express the scale dependence of a renormalized coupling. It is defined as the value of the coupling at a renormalization scale that is smaller by a factor of two

$$
\left.\sigma(u) \equiv \bar{g}^{2}(\mu / 2)\right|_{\bar{g}^{2}(\mu)=u} .
$$

Both are related by

$$
-\ln 2=\int_{\sqrt{u}}^{\sqrt{\sigma(u)}} \frac{d x}{\beta(x)} .
$$

The step-scaling function is more directly accessible through computer simulations and plays a central role in our calculation. Its perturbative expansion is

$$
\begin{aligned}
\sigma(u) & =u+s_{0} u^{2}+s_{1} u^{3}+s_{2} u^{4}+\ldots \\
s_{0} & =2 b_{0} \ln 2, \quad s_{1}=\left(2 b_{0} \ln 2\right)^{2}+2 b_{1} \ln 2, \quad \text { etc. }
\end{aligned}
$$

\section{Strategy}

Lattice QCD is used as a non-perturbative tool that allows to determine the $\Lambda$ parameter of QCD from low energy experiments.

Our computational strategy can be summarized as follows. In large volume QCD simulations the value of a hadronic quantity, like the pion or kaon decay constant (or, in our case, a linear combination of both, denoted by $f_{\pi K}$ ), is determined at the physical mass point in lattice units on lattices with different lattice spacings. The experimental measurement of the same quantity ${ }^{2}$ allows to map out a relationship between the bare coupling $g_{0}$ of the chosen lattice action and the lattice spacing in fm. At this point one can switch to massless small volume simulations with a known box size $L_{\text {had }}$. A renormalized coupling $\bar{g}(\mu)$ in a finite volume scheme is defined, in which the renormalization scale is tied to the linear box size $\mu=L^{-1}$. The $\beta$ function of this coupling is determined non-perturbatively for a wide range of couplings, by simulations of lattices with decreasing box sizes. Once the $\beta$ function is known, it is used to determine the value of the renormalized coupling at a high energy scale $\mu=\mu_{\mathrm{PT}} \approx 70 \mathrm{GeV}$, provided that its value at $\mu \equiv L_{\text {had }}^{-1}$ is known. At these high energies the coupling is small and perturbation theory becomes reliable and is used to relate the coupling to the $\Lambda$ parameter, i.e. a perturbative approximation of $\beta$ is used in Eq. (5). This can then be translated exactly into the $\Lambda$ parameter of a more common scheme. All of this is carried out in massive $\left(N_{\mathrm{f}}=2+1\right.$, large volume) or massless $\left(N_{\mathrm{f}}=3\right.$, finite volume) QCD. To obtain the $\Lambda$ parameter in four, five or six flavor QCD, perturbative decoupling [21-26] is invoked.

In practice the above procedure is extended by two steps. The first is to use two different finite volume schemes, each offering significant advantages in the energy region where they are utilized. The couplings of these two schemes are matched non-perturbatively at an intermediate scale $L_{0}^{-1} \approx 5 \mathrm{GeV}$. The other step is to use the flow scale $t_{0}$ [27] as an intermediate scale during the scale-setting, i.e. to first determine the dimensionless combination of $t_{0}$ and $f_{\pi K}$ and then the ratio $L_{\text {had }} / \sqrt{t_{0}}$. In total the calculation can be summarized by

$$
\Lambda_{\overline{\mathrm{MS}}}^{(3)}=\underbrace{\frac{f_{\pi K}^{\mathrm{PDG}}}{f_{\pi K} \sqrt{t_{0}}}}_{\begin{array}{c}
\text { scale setting } \\
\text { Sec. } 4
\end{array}} \times \underbrace{\frac{\sqrt{t_{0}}}{L_{\mathrm{had}}}}_{\begin{array}{c}
\text { connection to } \\
\text { Sec. } 5.5
\end{array}} \times \underbrace{\frac{L_{\mathrm{had}}}{2 L_{0}}}_{\begin{array}{c}
\text { scheme } 1 \\
\text { Sec. 5.3 }
\end{array}} \times \underbrace{\frac{2 L_{0}}{L_{0}}}_{\begin{array}{c}
\text { change of schemes } \\
\text { Sec. 5.4 }
\end{array}} \times \underbrace{\Lambda_{\mathrm{MS}}^{(3)} L_{0}}_{\begin{array}{c}
\text { scheme } 2 \\
\text { Sec. 5.2 }
\end{array}},
$$

\footnotetext{
${ }^{2}$ In pure QCD $f_{\pi}$ is defined by a matrix element of the axial current between the vacuum and the pion state. Relating this to the experimentally measured values is intricate [20] and to some extent convention dependent.
} 
where each factor is largely independent from the others.

\section{Scale Setting}

The experimental inputs needed for the determination of the $\Lambda$-parameter enter the computation through the process of scale-setting. I.e. the relation between the bare coupling $g_{0}$ and the lattice spacing in fm depends on these inputs, for which we take

$$
\begin{aligned}
f_{\pi K}^{\text {phys }} \equiv \frac{2}{3} f_{K}^{\text {phys }}+\frac{1}{3} f_{\pi}^{\text {phys }} & =147.6(5) \mathrm{MeV}, \\
m_{\pi}^{\text {phys }} & =134.8(3) \mathrm{MeV}, \\
m_{K}^{\text {phys }} & =494.2(3) \mathrm{MeV} .
\end{aligned}
$$

The meson masses, corrected for isospin breaking and electro-magnetic effects, are taken from FLAG [2], the decay constants from the PDG [28].

The large-volume simulations were carried out within the "Coordinated Lattice Simulations" consortium (CLS) [9]. The set of ensembles was generated using a tree-level Symanzik improved gauge action [29] and 2+1 flavors of non-perturbatively clover improved [30, 31] Wilson fermions. Open boundary conditions in temporal directions made simulations at small lattice spacings, down to $a \approx 0.039 \mathrm{fm}$ possible, without facing problems due to topological critical slowing down [32, 33]. All simulations were carried out using the openQCD simulation suite ${ }^{3}$ [34].

Finite lattice spacings and unphysical quark masses make a chiral-continuum extrapolation necessary before the scale can be set. It is convenient to use the flow scale $t_{0}$ [27] as an intermediate scale and to measure

$$
\begin{array}{rlr}
8 t_{0} m_{\pi}^{2} & \equiv \phi_{2} & \sim m_{\mathrm{up}} \\
8 t_{0}\left(m_{K}^{2}+\frac{m_{\pi}^{2}}{2}\right) & \equiv \phi_{4} & \sim m_{\mathrm{up}}+m_{\text {down }}+m_{\text {strange }} \\
\sqrt{t_{0}} f_{\pi K} & & \\
t_{0} / a^{2} & &
\end{array}
$$

on all ensembles. For a precise definition of these observables, bare parameters and choices of plateau regions, we refer the reader to [10]. The parameters of the CLS [9] ensembles were chosen such that $\phi_{4}$ is approximately constant and close to its physical value. Values of $\phi_{2}$ span a range corresponding to $200 \mathrm{MeV} \lesssim m_{\pi} \lesssim 420 \mathrm{MeV}$. The simulated bare couplings correspond to four different lattice spacings between $0.04 \mathrm{fm}$ and $0.09 \mathrm{fm}$. For the dependence of $\sqrt{t_{0}} f_{\pi K}$ on $\phi_{2}$ and the lattice spacing, different assumptions can be made. A Taylor expansion around the flavor-symmetric point motivates the ansatz [35]

$$
f^{\text {Taylor }}\left(\phi_{2}, a\right)=c_{0}+c_{1}\left(\phi_{2}-\phi_{2}^{\mathrm{sym}}\right)^{2}+c_{2} \frac{a^{2}}{t_{0}^{\mathrm{sym}}},
$$

while chiral perturbation theory $[36,37]$ suggests

$$
f^{\chi \mathrm{PT}}\left(\phi_{2}, a\right)=\left(\sqrt{t_{0}} f_{\pi K}\right)^{\mathrm{sym}}\left[1-\frac{7\left(L_{\pi}-L_{\pi}^{\mathrm{sym}}\right)}{6}-\frac{4\left(L_{K}-L_{K}^{\mathrm{sym}}\right)}{3}-\frac{L_{\eta}-L_{\eta}^{\mathrm{sym}}}{2}\right]+c_{4} \frac{a^{2}}{t_{0}^{\mathrm{sym}}},
$$

\footnotetext{
${ }^{3}$ http://luscher.web.cern.ch/luscher/openQCD/
} 
with logarithms $L_{x}=\frac{m_{x}^{2}}{(4 \pi f)^{2}} \ln \left[\frac{m_{x}^{2}}{(4 \pi f)^{2}}\right]$. These and other functions can be used to read off the value at the physical point. The intermediate scale $\sqrt{t_{0}^{\text {phys }}}$ in fm is given by $\sqrt{t_{0}^{\text {phys }}}=f\left(\phi_{2}^{\text {phys }}, 0\right) / f_{\pi K}^{\text {phys }}$. The lattice spacings in fm then follow from the $t_{0} / a^{2}$ measurements, extrapolated to $\phi_{2}=\phi_{2}^{\text {phys }}$.

The value of $t_{0}^{\text {phys }}$ that was used to plan the simulations and fix the chiral trajectory was slightly different than the final result of the procedure above. And even if not, the tuning of the ensembles only has a finite precision. Small corrections of the mis-tuning and small changes of the target values are necessary. They can be carried out, if the derivatives of all observables Eq. (16)-Eq. (19) with respect to the bare masses are known. These derivatives are measured as described in [10] and corrections

$$
O\left(m_{0}^{\prime}\right)=O\left(m_{0}\right)+\left(m_{0}^{\prime}-m_{0}\right) \frac{d O}{d m_{0}}+O\left(\left(m_{0}^{\prime}-m_{0}\right)^{2}\right)
$$

are made until a fixed point in the value of $t_{0}^{\text {phys }}$ is found. Fig. 2 shows two of the chiral-continuum extrapolations that were attempted after all ensembles were mass-shifted such that $\phi_{4}=\phi_{4}^{\text {phys }}$.
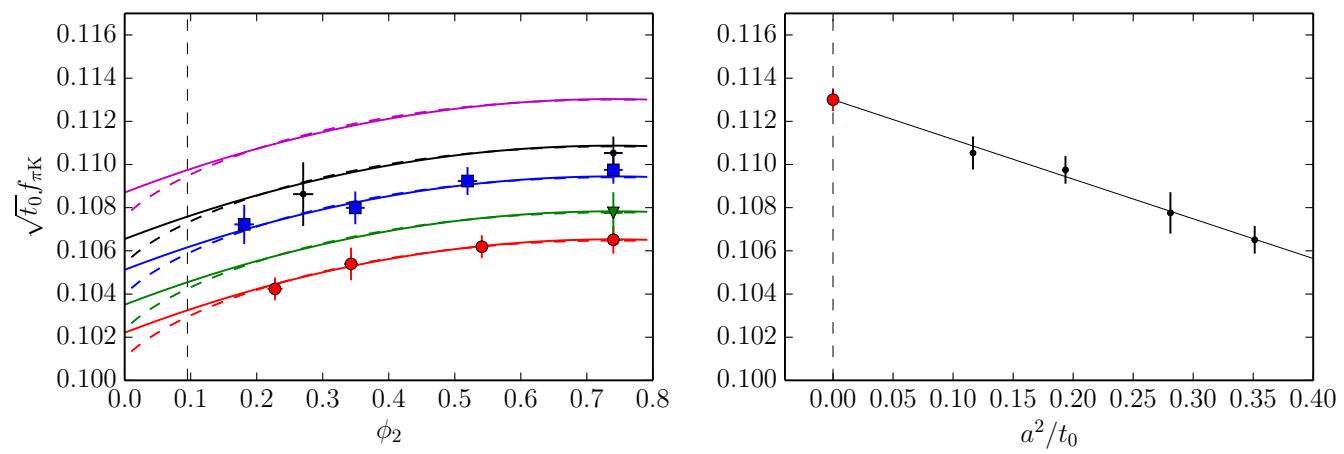

Figure 2. The left panel shows chiral and continuum extrapolations of $\sqrt{t_{0}} f_{\pi K}$. Solid lines correspond to extrapolations according to Eq. (20) and dashed lines to Eq. (21). From bottom to top the lattice spacing decreases, the corresponding bare couplings $6 / g_{0}^{2}$ are 3.4 (red), 3.46 (green), 3.55 (blue) and 3.7 (black). The extrapolated continuum curve (magenta) is on top. In the right panel it is shown, how well the global fit describes the lattice spacing dependence at the flavor symmetric mass point, where data are available for all lattice spacings.

A further improvement of the procedure above is to replace $\sqrt{t_{0}} f_{\pi K}$ by $\sqrt{t_{0}^{\star}} f_{\pi K}$, where $t_{0}^{\star}$ is defined on the unphysical mass-point where $m_{\mathrm{up}}=m_{\text {down }}=m_{\text {strange }}$ and $\phi_{4}=1.11$. The advantages are that at this mass-point simulations are comparatively cheap, no chiral extrapolations of $t_{0} / a^{2}$ are necessary, finite volume effects are smaller and, by definition, the mass-point remains unchanged, even if in the future, with higher statistics, the values of $\phi_{2}^{\text {phys }}$ and $\phi_{4}^{\text {phys }}$ should change.

Based on the CLS ensembles the procedure sketched above leads to

$$
\sqrt{8 t_{0}^{\star}}=0.413(5)(2) \mathrm{fm} .
$$

The first error is statistical, the second accounts for uncertainties related to the chiral extrapolations. Different functional forms have been tried and subsets of data neglected/included in order to asses its size [10]. The resulting lattice spacings are summarized in Tab. 1. 
Table 1. Bare couplings and the corresponding lattice spacings of the CLS ensembles.

\begin{tabular}{lll}
\hline $6 / g_{0}^{2}$ & $t_{0}^{\star} / a^{2}$ & $a$ in fm \\
\hline 3.40 & $2.862(6)$ & 0.086 \\
3.46 & $3.662(13)$ & 0.076 \\
3.55 & $5.166(18)$ & 0.064 \\
3.70 & $8.596(31)$ & 0.050 \\
3.85 & $14.036(57)$ & 0.039 \\
\hline
\end{tabular}

Table 2. Bare couplings such that $\bar{g}_{\mathrm{GF}}^{2}\left(L_{\mathrm{had}}^{-1}\right)=11.31$ for various $L_{\mathrm{had}} / a$.

\begin{tabular}{ll}
\hline $6 / g_{0}^{2}$ & $L_{\mathrm{had}} / a$ \\
\hline 3.3998 & $12.000(58)$ \\
3.5498 & $16.000(30)$ \\
3.6867 & $20.000(83)$ \\
3.8000 & $24.000(105)$ \\
3.9791 & $32.000(153)$ \\
\hline
\end{tabular}

\section{Running}

In finite volume renormalization schemes the renormalization scale is tied to the linear box size of the world $\mu \equiv L^{-1}$. To determine the $\beta$ or step-scaling function numerically, a sequence of simulations is necessary. For instance, to compute $\sigma(u)$ the necessary steps are sketched in Fig. 3. First a lattice reso-

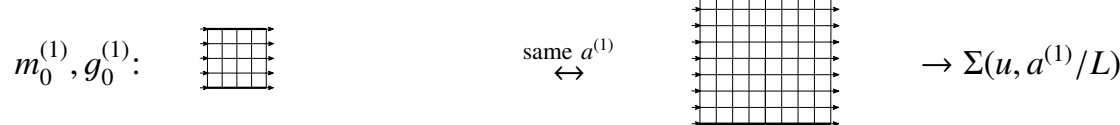

$$
\begin{aligned}
& £ \text { same } L, \bar{g}^{2}\left(L^{-1}\right) \\
& \begin{aligned}
m_{0}^{(2)}, g_{0}^{(2)}: & \\
& \downarrow \text { same } L, \bar{g}^{2}\left(L^{-1}\right)
\end{aligned} \\
& m_{0}^{(3)}, g_{0}^{(3)}: \quad \stackrel{\text { same } a^{(3)}}{\leftrightarrow} \\
& \bar{g}^{2}=u, \bar{m}=0 \\
& \rightarrow \Sigma\left(u, a^{(2)} / L\right) \\
& \rightarrow \Sigma\left(u, a^{(3)} / L\right) \\
& \downarrow \text { cont. limit }
\end{aligned}
$$
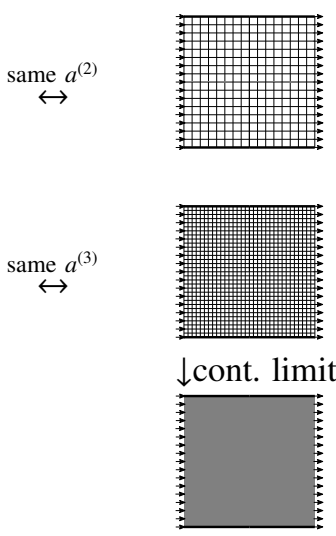

Figure 3. A sketch of the steps necessary to compute one continuum value of a step-scaling function $\sigma$.

lution $L / a$ is chosen. Then the bare coupling $g_{0}$ and the bare mass $m_{0}$ are tuned such that renormalized masses are zero, and the renormalized coupling $\bar{g}^{2}=u$. With the same bare parameters another simulation with $2 L / a$ lattice points in each direction is carried out. Up to lattice artifacts, same $g_{0}$ implies same lattice spacing, so the box-size is doubled (or the renormalization scale halved). Measuring the renormalized coupling on the doubled lattice yields a lattice estimate of the step-scaling function $\Sigma(u, a / L)$. This has to be repeated with the same $u$, but finer lattice resolutions, so that a continuum limit can be taken $\sigma(u)=\lim _{a / L \rightarrow 0} \Sigma(u, a / L)$. Finally all of this has to be repeated at various values of $u$, such that a safe interpolation to arbitrary $u$ values becomes possible. 
Instead of separate continuum extrapolations for each $u$ and subsequent parametrization of $\sigma(u)$, both can be combined into one step by performing a global fit. A possible ansatz is for instance

$$
\Sigma(u, a / L)=u+u^{2} \sum_{k=0}^{n_{\sigma}} s_{k} u^{k}+\left(\frac{a}{L}\right)^{2} \sum_{k=1}^{n_{\rho}} \rho_{k} u^{k+i+1}
$$

with the lowest $s_{k}$ (up to $s_{1}$ or $s_{2}$ ) taken from perturbation theory and the higher left as fit parameters. Leading lattice artifacts are parametrized by the coefficients $\rho_{k}$ and the value of $i$ may be zero, or higher, depending on the order in perturbation theory to which the lattice artifacts were removed from the raw data. Different combinations of orders of polynomials $\left(n_{\sigma}, n_{\rho}\right)$ can be tried.

A somewhat different approach to fit the data is to choose a parametrization of the $\beta$-function e.g.

$$
\begin{aligned}
& \beta(g)=-g^{3}\left(b_{0}+b_{1} g^{2}+b_{2} g^{4}+\ldots\right), \quad \text { or } \\
& \beta(g)=-\frac{g^{3}}{p_{0}+p_{1} g^{2}+p_{2} g^{4}+\ldots}
\end{aligned}
$$

and insert it into Eq. (9). In particular the second choice is interesting, because it leads to a fit that is linear in the fit parameters $p_{k}$ [12]. Making again an ansatz for the leading lattice artifacts the fit would minimize the violations of the equation

$$
\begin{aligned}
\ln 2= & -\frac{p_{0}}{2}\left[\frac{1}{\Sigma(u, a / L)}-\frac{1}{u}\right]+\frac{p_{1}}{2} \ln \left[\frac{\Sigma(u, a / L)}{u}\right]+\sum_{n=1}^{n_{p}} \frac{p_{n+1}}{2 n}\left[\Sigma^{n}(u, a / L)-u^{n}\right] \\
& +\left(\frac{a}{L}\right)^{2} \sum_{k=1}^{n_{\rho}} \rho_{k} u^{k+i+1}
\end{aligned}
$$

where $\Sigma(u, a / L)$ are the data.

The first choice leads to a non-linear fit, which is feasible if one can get away with very few parameters $b_{3}, b_{4}, \ldots$ and take $b_{0}, b_{1}, b_{2}$ from perturbation theory.

\subsection{QCD Schrödinger-Functional}

Boundary conditions play a crucial role for finite volume schemes. Depending on the choice simulations with massless fermions become more or less expensive, or not possible at all. Schrödinger Functional (SF) boundaries are particularly interesting. They induce a solid spectral gap in the Dirac operator [38], allow to define useful boundary-to-bulk and boundary-to-boundary correlation functions $[39,40]$ and can be used to define finite volume couplings with very good properties.

SF boundaries consist of Dirichlet boundaries in time for both fermion and gauge fields, and periodic (up to a phase) boundaries in spacial directions. Gauge fields satisfy

$$
\left.U_{k}(x)\right|_{x_{0}=0}=\exp \left(a C_{k}\right),\left.\quad U_{k}(x)\right|_{x_{0}=T}=\exp \left(a C_{k}^{\prime}\right), \quad U_{\mu}(x+L \hat{k})=U_{\mu}(x) .
$$

For the choice of boundary gauge fields we restrict ourselves to Abelian SU(3) matrices parametrized by the angles $\eta$ and $v[41]$,

$$
C_{k}=\frac{i}{L}\left(\begin{array}{lll}
\eta-\frac{\pi}{3} & & \\
& \eta v-\frac{\eta}{2} & \\
& & -\eta v-\frac{\eta}{2}+\frac{\pi}{3}
\end{array}\right), \quad C_{k}^{\prime}=\frac{i}{L}\left(\begin{array}{lll}
-\eta-\pi & \\
& \eta v+\frac{\eta}{2}+\frac{\pi}{3} & \\
& & \frac{\eta}{2}-\eta v+\frac{2 \pi}{3}
\end{array}\right)
$$

For matter fields conditions

$$
\left.\frac{1}{2}\left[\mathbb{1}-\gamma_{0}\right] \psi(x)\right|_{x_{0}=0}=0,\left.\quad \frac{1}{2}\left[\mathbb{1}+\gamma_{0}\right] \psi(x)\right|_{x_{0}=T}=0, \quad \psi(x+L \hat{k})=e^{i \theta} \psi(x),
$$

and similarly for $\bar{\psi}$ are imposed [42]. 


\subsection{Schrödinger-Functional Coupling}

The standard definition of a SF-coupling $[41,43]$ is based on the sensitivity of the effective action

$$
\Gamma=\ln \left[\int D[U, \bar{\psi}, \psi] e^{-S[U, \bar{\psi}, \psi]}\right]
$$

to the change of boundaries

$$
\bar{g}_{v} \equiv k\left[\left.\frac{\partial \Gamma}{\partial \eta}\right|_{\eta=0}\right]^{-1} .
$$

This is in fact a whole family of renormalized couplings, the most frequently used one being $\bar{g}_{\mathrm{SF}} \equiv$ $\bar{g}_{v=0}$. The normalization $k$ is chosen such that $\bar{g}_{\mathrm{SF}}^{2}=g_{0}^{2}+O\left(g_{0}^{4}\right)$, [43].

The advantages of coupling Eq. (32) are a good statistical precision, remarkably small lattice artifacts, relatively small computational costs and a good theoretical understanding $[19,38]$. Its $\beta$ function is known to three loops. In consequence it has been successfully used for the last quarter century to compute $\Lambda$-parameters of $N_{\mathrm{f}}=0$ [43], $N_{\mathrm{f}}=2$ [44], $N_{\mathrm{f}}=3$ [4] and $N_{\mathrm{f}}=4$ [45] QCD and in various beyond-the-standard-model applications, mainly related to a composite Higgs, see e.g. [46] and references therein.

The main disadvantage is that the statistical precision deteriorates at low energies and close to the continuum limit. To leading order, the relative error of $\bar{g}_{\mathrm{SF}}^{2}$ is proportional to $\bar{g}_{\mathrm{SF}}^{2}$, which makes this scheme particularly useful at higher energies, but problematic when $\bar{g}_{\mathrm{SF}}^{2}$ is large. Moreover the variance of $\frac{\partial \Gamma}{\partial \eta}$ is not a renormalized quantity. It diverges in the continuum limit [47], which further increases the costs of the already expensive fine lattices. Here, the SF scheme is used at high energies $\gtrsim 5 \mathrm{GeV}$ only.

The step-scaling function of the SF coupling is computed as described in Sec. 5 for couplings $u \in\{1.1089,1.1845,1.2657,1.3627,1.4808,1.6173,1.7943,2.012\}$, on lattices with $L / a \in\{4,6,8,12\}$ (and the corresponding doubled lattices). $L / a=4$ lattices were excluded from the final analysis, $L / a=12$ lattices are available only for a subset of the couplings. The largest coupling implicitly defines the scale $L_{0}$ at which finite volume schemes are switched

$$
\bar{g}_{\mathrm{SF}}^{2}\left(L_{0}^{-1}\right) \equiv 2.012 .
$$

To be able to make most use of available perturbative results, the lattice action for this part of the project is Wilson's plaquette action with clover-improved Wilson fermions. For this setup the coefficient of the clover term is known non-perturbatively [48] and the boundary improvement coefficients $c_{t}$ [41] and $\tilde{c}_{t}$ [49] are known to two [19] and one [50] loop respectively. An error due to the limited knowledge of $c_{t}$ and $\tilde{c}_{t}$ is propagated onto our data and perturbative lattice artifacts are subtracted as detailed in [11], before trying various fitting procedures.

The left panel of Fig. 4 shows our data and two possible continuum extrapolations, namely Eq. (24) with $n_{\sigma}=3, i=2$ and $n_{\rho}=2$ or 0 .

With the non-perturbatively determined $\beta$ or step-scaling function at hand it is possible to determine the coupling at renormalization scales $L_{0}^{-1} \rightarrow 2 L_{0}^{-1} \rightarrow 4 L_{0}^{-1} \ldots$. At step $n$ Eq. (5) can be used to obtain an estimate of $2^{-n} L_{0} \Lambda^{(3)}$ with a perturbative truncation error, that decreases with growing $n$. The situation is depicted in Fig. 8. The final result of this part is (see [11] for further details)

$$
L_{0} \Lambda_{\mathrm{SF}}^{(3)}=0.0303(8), \quad \text { or } \quad L_{0} \Lambda_{\mathrm{MS}}^{(3)}=0.0791(21) .
$$

The well known relation [38] between the SF and the $\overline{\mathrm{MS}}$ schemes $\Lambda_{\mathrm{SF}}^{(3)}=0.38286(2) \Lambda_{\overline{\mathrm{MS}}}^{(3)}$ is used here. 


\subsection{Gradient Flow Coupling}

A recently significantly improved [27, 51-57] understanding of the Yang-Mills gradient flow (GF) [58] has opened the way for the definition of novel scales like $t_{0}$ [27] or $w_{0}$ [59] and for the introduction of new renormalization schemes [60-62].

Correlation functions of fields $B_{\mu}$, that are solutions of the gradient flow equation

$$
\begin{aligned}
\partial_{t} B_{\mu}(t, x) & =D_{v} G_{v \mu}(t, x), \quad B_{\mu}(0, x)=A_{\mu}(x), \\
D_{\mu} & =\partial_{\mu}+\left[B_{\mu}, \cdot\right], \\
G_{\mu \nu} & =\partial_{\mu} B_{v}-\partial_{v} B_{\mu}+\left[B_{\mu}, B_{v}\right]
\end{aligned}
$$

were found to be automatically renormalized at flow times $t>0$. Simple gauge-invariant combinations, like the action density, can be used in the definitions of couplings and scales, which can then be measured extremely precisely. Moreover their variances are renormalized quantities themselves, such that the continuum limit can be approached without the problem of a diverging noise to signal ratio.

Already in [27] the proposal was made to define a renormalized coupling based on the dimensionless combination $t^{2}\left\langle G_{\mu \nu}^{a} G_{\mu \nu}^{a}\right\rangle$, where the renormalization scale is given by the flow time $\mu=1 / \sqrt{8 t}$. This original gradient flow renormalization scheme has been recently studied to 2 loops in perturbation theory [57]. Different finite volume renormalization schemes, where the flow time (and therefore renormalization scale) are tied to the box size $\mu=1 / L=c / \sqrt{8 t}$, were invented $[60,61,63]$ and successfully applied [64-68]. The variant that is used in this work follows [61]. The running coupling is defined to be

$$
\bar{g}_{\mathrm{GF}}^{2}(\mu)=\left.\mathcal{N}^{-1} \frac{t^{2}}{4} \frac{\left\langle G_{\mu \nu}^{a}(t, x) G_{\mu \nu}^{a}(t, x) \delta_{Q, 0}\right\rangle}{\left\langle\delta_{Q, 0}\right\rangle}\right|_{\sqrt{8 t=c L, x_{0}=T / 2}},
$$

where $L=T$ is the size of a massless Schrödinger functional without background field $\left(C=C^{\prime}=\right.$ $0), c=0.3, \mathcal{N}$ a computable normalization factor and the summation over $\mu$ and $v$ is restricted to the spacial components only. $Q=\frac{1}{32 \pi^{2}} \int d^{4} x \epsilon_{\mu \nu \rho \sigma} G_{\mu \nu}^{a}(t, x) G_{\rho \sigma}^{a}(t, x)$ is the topological charge and a projection onto the trivial sector is included in the definition Eq. (35). This projection reduces the variance and simplifies the error analysis in cases where topological sectors are sampled very slowly [32]. Moreover algorithms can be used, that deliberately stay in the trivial sector [12].

On the lattice a discretization has to be chosen for the action, for the flow equation and for the definition of the observable $G_{\mu \nu}^{a}(t, x) G_{\mu \nu}^{a}(t, x)$. In order to be able to determine the largest simulated box size $L_{\text {had }}$ in fm, defined implicitly by

$$
\bar{g}_{\mathrm{GF}}^{2}\left(L_{\mathrm{had}}^{-1}\right) \equiv 11.31,
$$

the discretization of the action needs to be the same as the one used by CLS, for which the scale was set. That means, a tree level Symanzik improved gauge action $\left(S_{\mathrm{LW}}\right)$ and massless clover improved Wilson fermions. In a finite volume it becomes necessary to specify, how exactly the SF boundary conditions are realized and which values for the boundary improvement coefficients $c_{t}$ and $\tilde{c}_{t}$ are used. We opt for choice B of ref. [69] for which the coefficients are known to one loop [70, 71].

For the discretization of the flow equation and the observable we follow [72] and use the Symazik $O\left(a^{2}\right)$ improved lattice flow equation, a.k.a "Zeuthen flow"

$$
a^{2}\left[\partial_{t} V_{\mu}(t, x)\right] V_{\mu}(t, x)^{\dagger}=-g_{0}^{2}\left(1+\frac{a^{2}}{12} \Delta_{\mu}\right) \partial_{x, \mu} S_{\mathrm{LW}}[V], \quad V_{\mu}(0, x)=U_{\mu}(x)
$$

Here $\partial_{x, \mu} S_{\mathrm{LW}}$ is the force derived from the improved action. For the observable we use the (at finite $t$ ) $O\left(a^{2}\right)$ improved choice that also enters $S_{\mathrm{LW}}$. 
Despite systematically removing several sources of $O\left(a^{2}\right)$ lattice artifacts, the remaining scaling violations are quite significant, especially when compared to those encountered in the SF scheme. Consequently, finer lattices were necessary for a controlled continuum extrapolation. The data set for this part of the project consists of lattices with $L / a \in\{8,12,16\}$ (and doubled lattices) at couplings $u \in$ $\{2.12,2.39,2.74,3.20,3.86,4.48,5.30,5.87,6.55\}$ (approximately). The right panel of Fig. 4 shows our data and two possible continuum extrapolations.
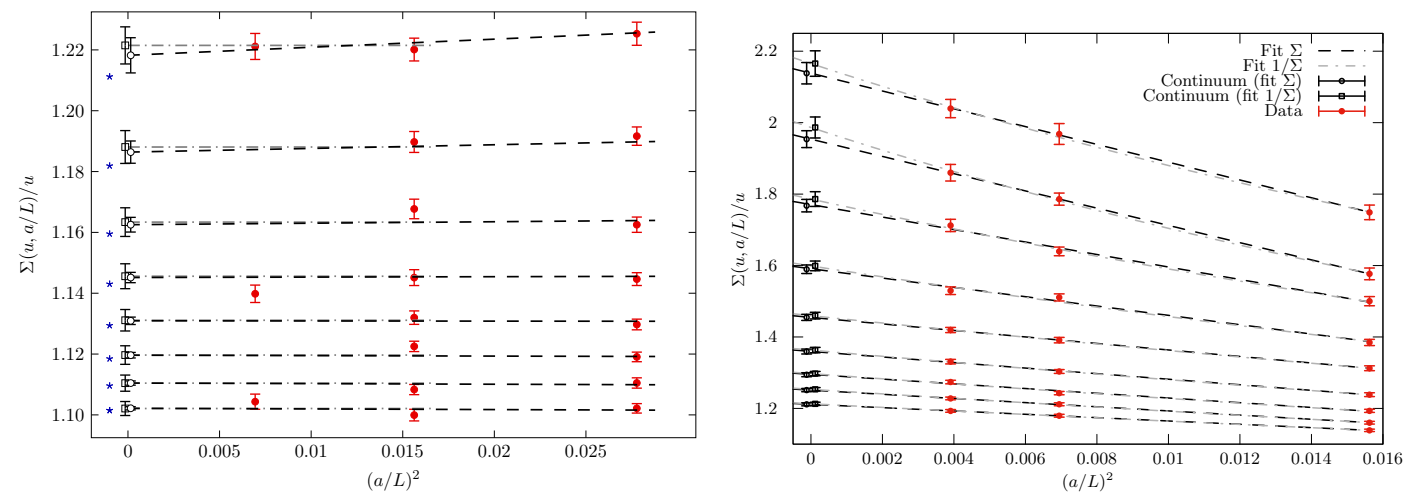

Figure 4. Exemplary continuum limits of the step-scaling functions in the SF (left) and the GF (right) schemes. Asterisks in the left plot mark perturbative predictions.

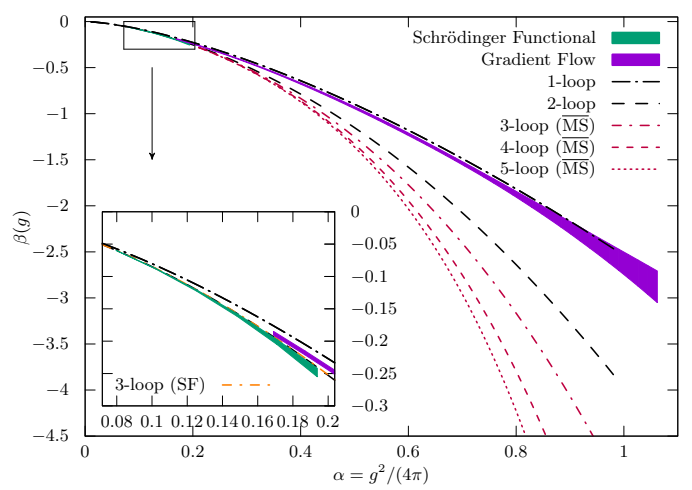

Figure 5. Non-perturbatively determined $\beta$-functions in the GF and SF schemes and a comparison to various perturbative orders.

Fig. 5 shows the non-perturbative continuum $\beta$ functions in both schemes in the range of couplings where they were determined. As expected, in the high energy regime the SF $\beta$-function follows the perturbative prediction quite closely. In the GF scheme, in the strong coupling region, the curve deviates noticeably from the two loop prediction. The running is slower, better (but not quite) described by the one loop expression. 


\subsection{Non-perturbative Matching}

The $\beta$ function of the GF scheme can be used to compute $L_{0}$ in $\mathrm{fm}$, if $L_{\text {had }}$ in $\mathrm{fm}$ is known. To do this, it is necessary to know the value of the coupling $\bar{g}_{\mathrm{GF}}^{2}\left(L_{0}^{-1}\right)$. This is not entirely trivial, because $L_{0}$ is defined implicitly in Eq. (33), i.e. using a different scheme with different boundary conditions and a different lattice action. Since $\bar{g}_{\mathrm{GF}}^{2}$ and $\bar{g}_{\mathrm{SF}}^{2}$ cannot be measured on the same ensembles a (small) set of new simulations is necessary. The scheme switch is combined with one iteration of step-scaling. For every $L / a \in\{12,16,24,32\}$ with $\bar{g}_{\mathrm{SF}}^{2}=2.012$ a simulation with the same action, same bare parameters, doubled linear lattice size and switched off boundary field is carried out. On these doubled lattices $\bar{g}_{\mathrm{GF}}^{2}\left(\left(2 L_{0}\right)^{-1}\right)$ is measured. Finally its continuum limit is obtained, as shown in Fig. 6. Its value, independent of the lattice action, is

$$
\bar{g}_{\mathrm{GF}}^{2}\left(\left(2 L_{0}\right)^{-1}\right)=2.6723(64)
$$

Inserting the numerically determined $\beta$-function into

$$
\ln \left[\frac{2 L_{0}}{L_{\mathrm{had}}}\right]=\int_{\bar{g}_{\mathrm{GF}}\left(\left(2 L_{0}\right)^{-1}\right)}^{\bar{g}_{\mathrm{GF}}\left(L_{\mathrm{had}}^{-1}\right)} \frac{d x}{\beta(x)}
$$

yields [12]

$$
L_{\text {had }} / L_{0}=21.86(42) \text {. }
$$

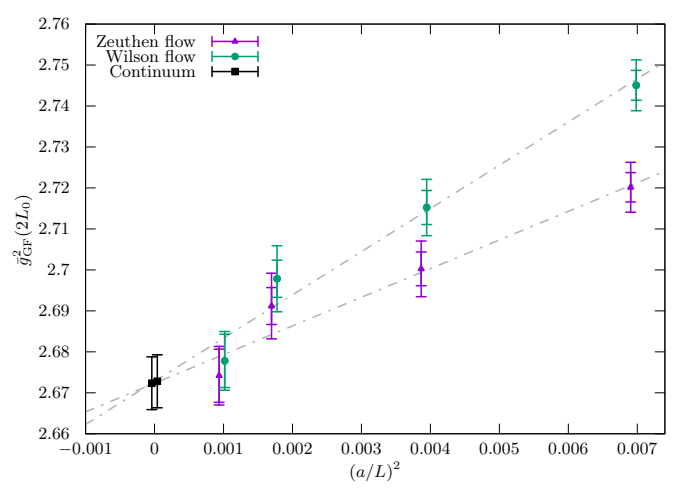

Figure 6. Non-perturbative matching of SF and GF couplings. Continuum extrapolations are shown for the standard (improved) definition of the GF coupling and for one in which the unimproved Wilson flow and an unimproved definition of the observable were used. The smaller error bars stem from $\bar{g}_{\mathrm{GF}}^{2}$, the complete error bar contains also the contribution due to a finite precision in the tuning to $\bar{g}_{\mathrm{SF}}^{2}=2.012$.

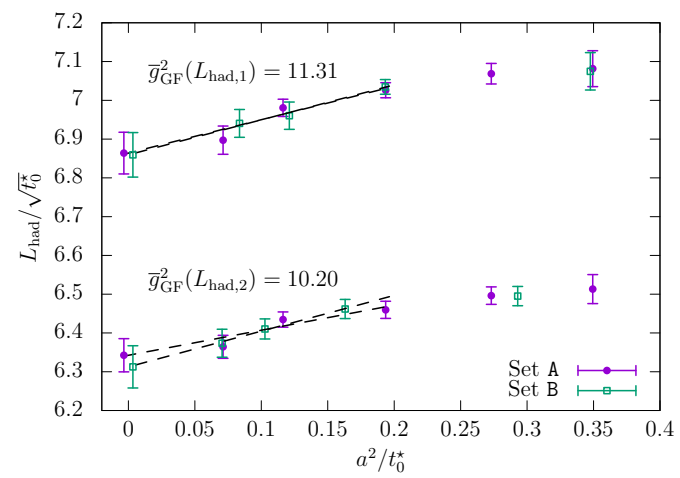

Figure 7. Determination of the continuum limit of the ratio $L_{\text {had }} / \sqrt{t_{0}^{\star}}$ for two definitions of $L_{\text {had }}$. In Set A the data in Tab. 2 are interpolated to the improved bare couplings corresponding to Tab. 1. In Set B the other way around. 


\subsection{Connection to Infinite Volume}

To combine everything into a final result according to Eq. (12), the last missing factor is $\frac{\sqrt{t_{0}}}{L_{\text {had }}}$ in the continuum limit. At finite lattice spacing the numerator is known in lattice units from Tab. 1. The denominator is known in lattice units as well - the bare couplings that result in $\bar{g}_{\mathrm{GF}}^{2}=11.31$ were found in subsection 5.3 for $L_{\mathrm{had}} / a \in\{8,12,16\}$. These, and in addition values of the bare coupling for $L_{\text {had }} / a \in\{20,24,32\}$ are summarized in Tab. 2 . The tuning has a finite precision and the associated error is propagated onto the listed $L_{\mathrm{had}} / a$ values.

The action for large volume and GF simulations is, apart from mass terms, the same, but the bare couplings in Tab. 1 differ from those in Tab. 2. In order for the lattice spacing to drop out in the ratio, the data in Tab. 1 need to be interpolated to the bare couplings of Tab. 2 or vice versa. Same $g_{0}$ means same $a$ up to $O(a)$ artifacts. These can be reduced to $O\left(a^{2}\right)$ if the bare couplings in Tab. 1 are replaced by $[49,73]$

$$
\tilde{g}_{0}^{2}=g_{0}^{2}\left(1+\frac{1}{3} \operatorname{tr}\left[a M_{q}\right] b_{g}\left(g_{0}\right)\right)
$$

$M_{q}$ is the subtracted quark mass matrix and $b_{g}$ an improvement coefficient, currently known only perturbatively [38] to one loop.

The interpolations are short and relatively simple. A polynomial in $6 / \tilde{g}_{0}^{2}$ is used as an ansatz to fit $\ln \left(\sqrt{a^{2} / t_{0}}\right)$ or $\ln \left(a / L_{\text {had }}\right)$. Details of the procedure can be found in the supplementary material of [13]. Once the interpolations are done, the continuum limit of the ratio can be taken as shown in Fig. 7. In addition to the standard definition of $L_{\text {had }}$ used so far, a slightly different choice corresponding to

$$
\bar{g}_{\mathrm{GF}}^{2}\left(L_{\mathrm{had}, 2}^{-1}\right)=10.2
$$

is shown in the figure. The complete analysis was carried out with both choices, in order to assess systematic effects induced by the interpolations.

Eq. (12) can now be evaluated. The result is

$$
\Lambda_{\overline{\mathrm{MS}}}^{(3)}=341(12) \mathrm{MeV} \text {. }
$$

\section{Perturbative Decoupling}

Eq. (43) is our main result. It is almost fully non-perturbative. Perturbation theory is only used at very small couplings $\alpha(\mu) \approx 0.1$ or $\mu \approx 70 \mathrm{GeV}$, where it can be trusted and was tested (Fig. 8).

For a comparison with experimental and other lattice determinations the $\Lambda$-parameter in theories with four, five or even six flavors is necessary. We obtain those values based on perturbative decoupling.

QCD with $N_{\mathrm{f}}$ massless quarks and one massive quark, with renormalized mass $\bar{m}$, can be described by an effective $N_{\mathrm{f}}$-flavor theory [74]. To leading order this effective theory is given by $N_{\mathrm{f}}$-flavor QCD. Subleading terms give rise to power corrections starting at $O\left(\Lambda^{2} / \bar{m}^{2}, E^{2} / \bar{m}^{2}\right)$. Up to such corrections, dimensionfull low energy $(E)$ quantities computed in the effective theory equal those in the $N_{\mathrm{f}}+1$ theory, if the coupling is matched

$$
\bar{g}^{\left(N_{\mathrm{f}}\right)}(\mu)=\bar{g}^{\left(N_{\mathrm{f}}+1\right)}(\mu) \times \xi\left(\bar{g}^{\left(N_{\mathrm{f}}+1\right)}(\mu), \bar{m} / \mu\right) .
$$

Through the matching $\bar{g}^{\left(N_{\mathrm{f}}\right)}(\mu)$ depends implicitly on $\bar{m}$. The matching function $\xi$ is known perturbatively in the $\overline{\mathrm{MS}}$-scheme to four loops [21-26]. The expansion assumes a particularly simple form if 
the renormalization scale is chosen to coincide with the scale $m^{*}$, defined such, that the running mass at scale $m^{*}$ equals $m^{*}$, i.e. $m^{*}=\bar{m}_{\overline{\mathrm{MS}}}\left(m^{*}\right)$. With this choice the coefficients in

$$
\xi(\bar{g}, 1)=1+c_{2} \bar{g}^{4}+c_{3} \bar{g}^{6}+c_{4} \bar{g}^{8}+O\left(\bar{g}^{10}\right)
$$

are pure numbers and $c_{1}$ is absent. Together with the perturbative $\beta$ function, this relation between couplings implies a relation between $\Lambda_{\overline{\mathrm{MS}}}^{\left(N_{\mathrm{f}}\right)}$ and $\Lambda_{\overline{\mathrm{MS}}}^{\left(N_{\mathrm{f}}+1\right)}$. Using as inputs $\Lambda_{\overline{\mathrm{MS}}}^{(3)}$ and $m_{\text {charm }}^{*}=1.280(25) \mathrm{GeV}$, $m_{\text {bottom }}^{*}=4.180(30) \mathrm{GeV}$, [1] and $m_{\text {top }}^{*}=165.9(2.2) \mathrm{GeV}$ [75] the four, five and six flavor $\Lambda$ parameters can be computed.

Two questions arise. How large are the neglected power corrections? Can perturbation theory be trusted at $\mu=m_{\text {charm }}^{*}$ ?

The first question has been addressed non-perturbatively in a simplified setup, where the decoupling was investigated for QCD with just two heavy quarks [76-78]. At the charm quark mass the power corrections in the investigated quantities were found to be tiny, typically around two permille. It is more difficult to give a definitive answer to the second question. It was addressed in [79] with encouraging results, i.e. that PT might be applicable for decoupling at the charm scale.

Here we can only test decoupling within perturbation theory itself. To do so, we translate our result for $\Lambda_{\overline{\mathrm{MS}}}^{(3)}$ to $\Lambda_{\overline{\mathrm{MS}}}^{(5)}$ and then, inverting Eq. (5), to $\alpha_{\overline{\mathrm{MS}}}^{(5)}\left(M_{\mathrm{Z}}\right)$, with $M_{\mathrm{Z}}=91.1876 \mathrm{GeV}$, [1]. This is done in perturbation theory with $n$ loop accuracy for the decoupling relations and $n+1$ loop accuracy for the $\beta$ function. The apparent convergence of the final result with $n$ is monitored and turns out to be excellent. The sequence is tabulated in Tab. 3. As a systematic error the difference between the $n=4$ and the $n=2$ result is taken, which, within perturbation theory, is conservative. It plays a minor role in the overall error budget.

Table 3. PT decoupling

\begin{tabular}{ccc}
\hline $\mathrm{n}$ (loops) & $\alpha_{\overline{\mathrm{MS}}}^{\left(N_{\mathrm{f}}=5\right)}$ & $\alpha_{n}-\alpha_{n-1}$ \\
\hline 1 & 0.11699 & \\
2 & 0.11827 & 0.00128 \\
3 & 0.11846 & 0.00019 \\
4 & 0.11852 & 0.00006 \\
\hline
\end{tabular}

\section{Conclusions}

Our final results are

$$
\begin{array}{rlrl}
\Lambda_{\overline{\mathrm{MS}}}^{(3)} & =341(12) \mathrm{MeV}, & \\
\Lambda_{\overline{\mathrm{MS}}}^{(4)} & =298(12)(03) \mathrm{MeV} & & \text { (pert. decoupling), } \\
\Lambda_{\overline{\mathrm{MS}}}^{(5)} & =215(10)(03) \mathrm{MeV} & & \text { (pert. decoupling), } \\
\Lambda_{\overline{\mathrm{MS}}}^{(6)} & =91.1(4.5)(1.3) \mathrm{MeV} & & \text { (pert. decoupling) } \\
\rightarrow \alpha_{\overline{\mathrm{MS}}}^{(5)}\left(m_{\mathrm{Z}}\right) & =0.1185(8)(3), & & \\
& 0.1174(16) & & \text { PDG non-lattice. }
\end{array}
$$

We reach a precision in $\alpha_{\mathrm{S}}$ of slightly below $0.7 \%$ which is very good - twice as precise as the nonlattice world average. The main sources of errors are the statistical errors of the step-scaling functions. 
They contribute $22 \%(\mathrm{GF})$ and $50 \%$ (SF) to the total relative error squared of $\alpha_{\mathrm{S}}$. The introduction of the second finite volume scheme, based on the gradient flow has paid off. The usually most problematic region of large couplings does not dominate the error as it did in the past. Observing the relatively large contribution to the error stemming from non-perturbative evolution of the SF coupling between 5 $\mathrm{GeV}$ and $70 \mathrm{GeV}$, the question arises whether one maybe could have used perturbation theory already at lower energies than we did. Fig. 8 shows the $\Lambda$ parameter in units of $L_{0}$, that one would obtain by

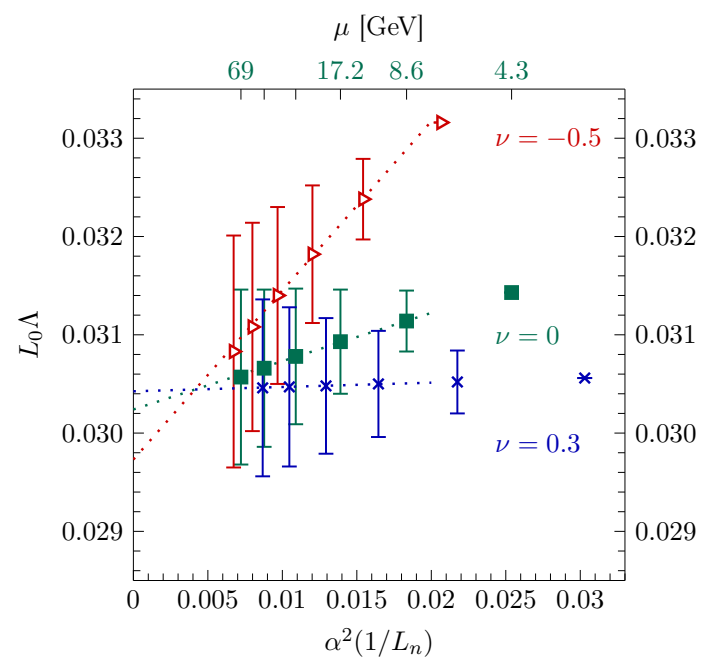

Figure 8. Results for $\Lambda$ parameters in different SF schemes, depending on the energy from which on three-loop perturbation theory was used.

using the perturbative 3-loop $\beta$-function starting from different energies. Purely perturbative behavior would show as a plateau in this plot. Although results starting from $\alpha \lesssim 0.14$ are compatible with each other, we see a drift of the central value. This linear behavior of $L_{0} \Lambda$ as a function of $\alpha^{2}$ can be explained by the presence of an effective $b_{3}$ term in the $\beta$-function. Relying on the 3-loop formula already at $\alpha \approx 0.14$ would reduce the statistical error significantly, but also introduce a systematic error of the order of $1 \sigma$. Such statements are highly scheme dependent. For instance Fig. 8 shows also the situation for two slightly different SF-couplings - the truncation error can become much worse, or completely absent, depending on the chosen scheme. The family of SF schemes considered here is generally believed to be particularly well behaved perturbatively. In other schemes truncation error could be much worse. An example is the $\alpha_{q q}$ scheme. Although its $\beta$-function is known to four loops, a significant deviation between perturbative and non-perturbative running can be observed at $\alpha_{q q} \approx 0.24$, [80].

For any future significant (e.g. factor $1 / 2$ in the error) improvements of our programme several challenging problems have to be overcome. The limited knowledge of boundary improvement coefficients will make pure $O\left(a^{2}\right)$ continuum extrapolations difficult. The subleading cutoff effects in $\bar{g}_{\mathrm{GF}}^{2}$ will become significant and will have to be addressed, for instance by considering even finer lattices than the $16^{4} \rightarrow 32^{4}$ ones used here. Electromagnetic and iso-spin effects in all quantities used for scale-setting will have to be accounted for. Finally, a fourth dynamical quark should better be con- 
sidered in order to shift the scale at which one relies on perturbative decoupling up to the bottom mass.

\section{Acknowledgments}

I would like to thank my colleagues in the ALPHA collaboration and in the CLS consortium for a pleasant and fruitful collaboration, in particular my co-authors in [9-13]. Francesco Knechtli and Rainer Sommer provided valuable comments on the manuscript for which I am deeply grateful.

We thank Pol Vilaseca for sharing his result on $\tilde{c}_{t}$ with us and Carlos Pena, Isabel Campos and David Preti for helping us generate several of the ensembles for the GF coupling.

The generation of large volume CLS ensembles would not have been possible without access to major computational resources. We acknowledge PRACE for awarding us access to the resource FERMI at CINECA and SuperMUC at the LRZ. We thank the Swiss National Supercomputing Centre for supporting us by a grant under project ID s384. We gratefully acknowledge the Gauss Centre for Supercomputing (GCS) for providing computing time through the John von Neumann Institute for Computing (NIC) on the GCS share of the supercomputer JUQUEEN at Jülich Supercomputing Centre (JSC). We thank the Helmhotz Institute Mainz and the University of Mainz for access to the "Clover" HPC Cluster. The generation of the finite volume ensembles required significant resources as well. We were granted computer time and support by HLRN (bep0040), by NIC at DESY, Zeuthen and on Altamira at IFCA for which we are grateful.

This work is based on previous work [81] supported strongly by the Deutsche Forschungsgemeinschaft in the SFB/TR 09.

\section{References}

[1] C. Patrignani et al. (Particle Data Group), Chin. Phys. C40, 100001 (2016)

[2] S. Aoki et al., Eur. Phys. J. C77, 112 (2017), 1607.00299

[3] K. Maltman, D. Leinweber, P. Moran, A. Sternbeck, Phys. Rev. D78, 114504 (2008), 0807.2020

[4] S. Aoki et al. (PACS-CS), JHEP 10, 053 (2009), 0906.3906

[5] C. McNeile, C.T.H. Davies, E. Follana, K. Hornbostel, G.P. Lepage, Phys. Rev. D82, 034512 (2010), 1004.4285

[6] A. Bazavov, N. Brambilla, X. Garcia i Tormo, P. Petreczky, J. Soto, A. Vairo, Phys. Rev. D90, 074038 (2014), 1407.8437

[7] B. Chakraborty, C.T.H. Davies, B. Galloway, P. Knecht, J. Koponen, G.C. Donald, R.J. Dowdall, G.P. Lepage, C. McNeile, Phys. Rev. D91, 054508 (2015), 1408. 4169

[8] M. Lüscher, P. Weisz, U. Wolff, Nucl. Phys. B359, 221 (1991)

[9] M. Bruno et al., JHEP 02, 043 (2015), 1411. 3982

[10] M. Bruno, T. Korzec, S. Schaefer, Phys. Rev. D95, 074504 (2017), 1608.08900

[11] M. Dalla Brida, P. Fritzsch, T. Korzec, A. Ramos, S. Sint, R. Sommer (ALPHA), Phys. Rev. Lett. 117, 182001 (2016), 1604.06193

[12] M. Dalla Brida, P. Fritzsch, T. Korzec, A. Ramos, S. Sint, R. Sommer (ALPHA), Phys. Rev. D95, 014507 (2017), 1607.06423

[13] M. Bruno, M. Dalla Brida, P. Fritzsch, T. Korzec, A. Ramos, S. Schaefer, H. Simma, S. Sint, R. Sommer (ALPHA), Phys. Rev. Lett. 119, 102001 (2017), 1706.03821

[14] T. van Ritbergen, J.A.M. Vermaseren, S.A. Larin, Phys. Lett. B400, 379 (1997), hep-ph/9701390 
[15] M. Czakon, Nucl. Phys. B710, 485 (2005), hep-ph/0411261

[16] P.A. Baikov, K.G. Chetyrkin, J.H. Kühn, Phys. Rev. Lett. 118, 082002 (2017), 1606.08659

[17] T. Luthe, A. Maier, P. Marquard, Y. Schröder, JHEP 07, 127 (2016), 1606.08662

[18] F. Herzog, B. Ruijl, T. Ueda, J.A.M. Vermaseren, A. Vogt, JHEP 02, 090 (2017), 1701.01404

[19] A. Bode, P. Weisz, U. Wolff (ALPHA), Nucl. Phys. B576, 517 (2000), [Erratum: Nucl. Phys.B600,453(2001)], hep-lat/9911018

[20] J. Gasser, G.R.S. Zarnauskas, Phys. Lett. B693, 122 (2010), 1008 . 3479

[21] S. Weinberg, Phys. Lett. 91B, 51 (1980)

[22] W. Bernreuther, W. Wetzel, Nucl. Phys. B197, 228 (1982), [Erratum: Nucl. Phys.B513,758(1998)]

[23] A.G. Grozin, M. Hoeschele, J. Hoff, M. Steinhauser, JHEP 09, 066 (2011), 1107 . 5970

[24] K.G. Chetyrkin, J.H. Kühn, C. Sturm, Nucl. Phys. B744, 121 (2006), hep-ph/0512060

[25] Y. Schröder, M. Steinhauser, JHEP 01, 051 (2006), hep-ph/0512058

[26] B.A. Kniehl, A.V. Kotikov, A.I. Onishchenko, O.L. Veretin, Phys. Rev. Lett. 97, 042001 (2006), hep-ph/0607202

[27] M. Lüscher, JHEP 08, 071 (2010), [Erratum: JHEP03,092(2014)], 1006. 4518

[28] K.A. Olive et al. (Particle Data Group), Chin. Phys. C38, 090001 (2014)

[29] M. Lüscher, P. Weisz, Commun. Math. Phys. 97, 59 (1985), [Erratum: Commun. Math. Phys.98,433(1985)]

[30] B. Sheikholeslami, R. Wohlert, Nucl. Phys. B259, 572 (1985)

[31] J. Bulava, S. Schaefer, Nucl. Phys. B874, 188 (2013), 1304.7093

[32] M. Lüscher, S. Schaefer, JHEP 07, 036 (2011), 1105 . 4749

[33] S. Schaefer, R. Sommer, F. Virotta (ALPHA), Nucl. Phys. B845, 93 (2011), 1009 . 5228

[34] M. Lüscher, S. Schaefer, Comput. Phys. Commun. 184, 519 (2013), 1206.2809

[35] W. Bietenholz et al., Phys. Lett. B690, 436 (2010), 1003. 1114

[36] O. Bär, M. Golterman, Phys. Rev. D89, 034505 (2014), [Erratum: Phys. Rev.D89,no.9,099905(2014)], 1312.4999

[37] J. Gasser, H. Leutwyler, Nucl. Phys. B250, 517 (1985)

[38] S. Sint, R. Sommer, Nucl. Phys. B465, 71 (1996), hep-lat/9508012

[39] K. Jansen, C. Liu, M. Lüscher, H. Simma, S. Sint, R. Sommer, P. Weisz, U. Wolff, Phys. Lett. B372, 275 (1996), hep-lat/9512009

[40] S. Capitani, M. Lüscher, R. Sommer, H. Wittig, Nucl. Phys. B544, 669 (1999), [Erratum: Nucl. Phys.B582,762(2000)], hep-lat/9810063

[41] M. Lüscher, R. Narayanan, P. Weisz, U. Wolff, Nucl. Phys. B384, 168 (1992), hep-lat/9207009

[42] S. Sint, Nucl. Phys. B421, 135 (1994), hep-lat/9312079

[43] M. Lüscher, R. Sommer, P. Weisz, U. Wolff, Nucl. Phys. B413, 481 (1994), hep-1at/9309005

[44] P. Fritzsch, F. Knechtli, B. Leder, M. Marinkovic, S. Schaefer, R. Sommer, F. Virotta, Nucl. Phys. B865, 397 (2012), 1205.5380

[45] F. Tekin, R. Sommer, U. Wolff (ALPHA), Nucl. Phys. B840, 114 (2010), 1006.0672

[46] C. Pica, PoS LATTICE2016, 015 (2016), 1701.07782

[47] G. de Divitiis, R. Frezzotti, M. Guagnelli, M. Lüscher, R. Petronzio, R. Sommer, P. Weisz, U. Wolff (Alpha), Nucl. Phys. B437, 447 (1995), hep-lat/9411017

[48] N. Yamada et al. (JLQCD, CP-PACS), Phys. Rev. D71, 054505 (2005), hep-lat/0406028

[49] M. Lüscher, S. Sint, R. Sommer, P. Weisz, Nucl. Phys. B478, 365 (1996), hep-lat/9605038 
[50] M. Lüscher, P. Weisz, Nucl. Phys. B479, 429 (1996), hep-lat/9606016

[51] R. Narayanan, H. Neuberger, JHEP 03, 064 (2006), hep-th/0601210

[52] M. Lüscher, P. Weisz, JHEP 02, 051 (2011), 1101.0963

[53] M. Lüscher, JHEP 04, 123 (2013), 1302 . 5246

[54] A. Shindler, Nucl. Phys. B881, 71 (2014), 1312.4908

[55] L. Del Debbio, A. Patella, A. Rago, JHEP 11, 212 (2013), 1306. 1173

[56] Z. Fodor, K. Holland, J. Kuti, S. Mondal, D. Nogradi, C.H. Wong, JHEP 09, 018 (2014), 1406.0827

[57] R.V. Harlander, T. Neumann, JHEP 06, 161 (2016), 1606.03756

[58] M.F. Atiyah, R. Bott, Phil. Trans. Roy. Soc. Lond. A308, 523 (1982)

[59] S. Borsanyi et al., JHEP 09, 010 (2012), 1203.4469

[60] Z. Fodor, K. Holland, J. Kuti, D. Nogradi, C.H. Wong, JHEP 11, 007 (2012), 1208 . 1051

[61] P. Fritzsch, A. Ramos, JHEP 10, 008 (2013), 1301 . 4388

[62] M. Lüscher, JHEP 06, 105 (2014), 1404.5930

[63] A. Ramos, JHEP 11, 101 (2014), 1409. 1445

[64] A. Hasenfratz, D. Schaich, A. Veernala, JHEP 06, 143 (2015), 1410. 5886

[65] Z. Fodor, K. Holland, J. Kuti, S. Mondal, D. Nogradi, C.H. Wong, JHEP 06, 019 (2015), 1503.01132

[66] J. Rantaharju, Phys. Rev. D93, 094516 (2016), 1512.02793

[67] Z. Fodor, K. Holland, J. Kuti, S. Mondal, D. Nogradi, C.H. Wong, Phys. Rev. D94, 091501 (2016), 1607.06121

[68] V. Leino, J. Rantaharju, T. Rantalaiho, K. Rummukainen, J.M. Suorsa, K. Tuominen, Phys. Rev. D95, 114516 (2017), 1701.04666

[69] S. Aoki, R. Frezzotti, P. Weisz, Nucl. Phys. B540, 501 (1999), hep-lat/9808007

[70] S. Takeda, S. Aoki, K. Ide, Phys. Rev. D68, 014505 (2003), hep-lat/0304013

[71] P. Vilaseca, Private communication, unpublished

[72] A. Ramos, S. Sint, Eur. Phys. J. C76, 15 (2016), 1508.05552

[73] T. Bhattacharya, R. Gupta, W. Lee, S.R. Sharpe, J.M.S. Wu, Phys. Rev. D73, 034504 (2006), hep-lat/0511014

[74] S. Weinberg, Phys. Rev. D8, 3497 (1973)

[75] J. Fuster, A. Irles, D. Melini, P. Uwer, M. Vos (2017), 1704.00540

[76] M. Bruno, J. Finkenrath, F. Knechtli, B. Leder, R. Sommer (ALPHA), Phys. Rev. Lett. 114, $102001(2015), 1410.8374$

[77] F. Knechtli, T. Korzec, B. Leder, G. Moir (2017), 1706. 04982

[78] F. Knechtli, T. Korzec, B. Leder, G. Moir, Decoupling of charm beyond leading order, 1710.07590

[79] F. Knechtli, M. Bruno, J. Finkenrath, B. Leder, R. Sommer (ALPHA), PoS LATTICE2015, 256 (2016), 1511.04914

[80] R. Sommer, M. Koren, N. Husung, P. Krah, SU(3) Yang Mills at small distances and fine lattices, in Proceedings, 35th International Symposium on Lattice Field Theory (Lattice2017): Granada, Spain, to appear in EPJ Web Conf.

[81] R. Sommer, U. Wolff, Nucl. Part. Phys. Proc. 261-262, 155 (2015), 1501.01861 